\title{
Refined numerical models for multidimensional type la supernova simulations
}

\author{
M. Reinecke, W. Hillebrandt, and J. C. Niemeyer \\ Max-Planck-Institut für Astrophysik, Karl-Schwarzschild-Str. 1, 85741 Garching, Germany
}

Received 15 October 2001 / Accepted 28 February 2002

\begin{abstract}
Following up on earlier work on this topic (Reinecke et al. 1999b,a), we present an improved set of numerical models for simulations of white dwarfs exploding as type Ia supernovae (SNe Ia). Two-dimensional simulations were used to test the reliability and numerical robustness of these algorithms; the results indicate that integral quantities like the total energy release are insensitive to changes of the grid resolution (above a certain threshold), which was not the case for our former code.

The models were further enhanced to allow fully three-dimensional simulations of SNe Ia. A direct comparison of a $2 \mathrm{D}$ and a $3 \mathrm{D}$ calculation with identical initial conditions shows that the explosion is considerably more energetic in three dimensions; this is most likely caused by the assumption of axisymmetry in $2 \mathrm{D}$, which inhibits the growth of flame instabilities in the azimuthal direction and thereby decreases the flame surface.
\end{abstract}

Key words. supernovae: general - hydrodynamics - turbulence - nuclear reactions, nucleosynthesis, abundances - methods: numerical

\section{Introduction}

The project described in this article is a direct continuation of the work presented by Reinecke et al. (1999b), and is therefore concerned with the accurate modeling of the rapid thermonuclear combustion processes which occur in a white dwarf during its explosion as a type Ia supernova. While the earlier results already gave several new insights into the explosion dynamics of such an event and its dependence on parameters like the ignition conditions, it nevertheless became clear that the numerical models employed at that time were not sufficient to reproduce characteristic properties of SNe Ia that are known from observations; most importantly, the total energy release predicted by the simulations lay consistently below the expectations. Meanwhile, several possible reasons for this deficiency could be identified and the models were enhanced and adjusted accordingly, leading to much more realistic results.

The following section gives details of the implemented changes and explains why they were required for the correct treatment of SNe Ia. These modifications affect the numerical description of the flame as well as the model for the unresolved turbulent motions and the thermonuclear reaction rates. Section 3 presents the results obtained from a two-dimensional resolution study, which is mainly intended to test the correctness and numerical robustness

\footnotetext{
Send offprint requests to: M. Reinecke,
} e-mail: martin@mpa-garching.mpg.de of the code, even though data obtained from 2D simulations is unlikely to agree with observations for reasons given in Sect. 5.

The first three-dimensional simulation performed with the new code is discussed in Sect. 4, with a focus on analyzing the similarities and differences compared to the axisymmetric 2D model.

Finally, in Sect. 5 our data are compared with observations and numerical simulations performed by other authors; furthermore, several possible future improvements of the code are presented briefly.

Forthcoming articles will focus on a more detailed analysis of three-dimensional simulations with varying initial conditions and numerical resolution.

\section{Improvements of the numerical models}

It must be kept in mind that the corrections and additional features introduced below aim at a more realistic description of the explosion dynamics and energetics. No effort was made to reproduce other aspects, like the exact chemical composition of the burned material, unless this was required for the goals mentioned above. As a consequence, the treatment of nuclear reactions might appear minimalistic in comparison to the large reaction networks typically used in one-dimensional simulations; but reaching a comparable level of detail in multidimensions would be prohibitively expensive and is fortunately not required for reliable predictions of the explosion strength. 


\subsection{Nuclear reactions}

In a $\mathrm{SN}$ Ia, the nuclear reactions can be distinguished depending on their time scales:

- The "fast" reactions, which transform the initial C/O mixture into nuclear statistic equilibium (NSE) at high densities or into a mixture of intermediate-mass elements at lower densities, take place at time scales $\tau_{\text {fast }}$ that are much shorter than the time $\tau_{\text {cross }}$ during which the flame crosses a grid cell:

$\tau_{\text {fast }} \ll \tau_{\text {cross }}=\Delta / s$,

where $\Delta$ denotes the linear extent of a cell and $s$ the flame propagation speed. Therefore the transition between unburned and burned material is too abrupt to be spatially resolved and should be modeled as a numerical discontinuity. This can be done by using the so-called level set technique (see Sect. 2.2);

- Composition changes due to the expansion of the star (i.e. changes of the NSE), on the other hand, are much slower than $\tau_{\text {cross }}$ and the Courant time step and can be modeled by simply changing the local species concentrations to the desired values after each time step.

This picture is not entirely correct, since there exist especially at lower densities - reactions behind the flame whose time scales are comparable to $\tau_{\text {cross }}$. However, these reactions have no significant effect on the internal energy or the molar mass of the material and therefore can be neglected if one is only interested in the hydrodynamics of the explosion.

The original version of the combustion algorithm (see Reinecke et al. 1999b) did not contain any slow reactions, and the fast reactions were approximated rather crudely by the instant fusion of carbon and oxygen to nickel. In the meantime we realized that such a description is oversimplified and cannot lead to accurate results in a type Ia model for several reasons:

- Combustion at high densities does not immediately produce nickel, but also a considerable fraction of $\alpha$ particles. The specific energy release of this reaction is much lower than for the production of pure nickel;

- However, as the density in the ashes drops due to the stellar bulk expansion, the equilibrium shifts towards the heavy elements and the energy "stored" in the $\alpha$ particles is released during the later explosion stages;

- As soon as the flame enters regions of intermediate density $\left(10^{7}-10^{8} \mathrm{~g} \mathrm{~cm}^{-3}\right)$, elements of intermediate mass are synthesized instead of nickel, and the specific energy release drops accordingly.

Since all of these formerly neglected phenomena may affect the explosion dynamics, they were implemented in our new scheme, which is described below:

- The initial mixture consists of ${ }^{12} \mathrm{C}$ and ${ }^{16} \mathrm{O}$ at low temperatures. Because of the electron degeneracy the fuel temperature is nearly decoupled from the rest of the thermodynamical quantities, and since temperature is not used to determine the initial reaction rates, its exact value is unimportant;

- When the flame passes through the fuel, carbon and oxygen are converted to ash, which has different compositions depending on the density of the unburned material. At high densities $\left(\rho>5.25 \times 10^{7} \mathrm{~g} \mathrm{~cm}^{-3}\right)$, a mixture of ${ }^{56} \mathrm{Ni}$ and $\alpha$-particles in nuclear statistic equilibrium is synthesized. Below that density burning only produces intermediate mass elements, which are represented by ${ }^{24} \mathrm{Mg}$. Once the density drops below $10^{7} \mathrm{~g} \mathrm{~cm}^{-3}$, no burning takes place;

- Because NSE is assumed in the ashes, the proportion of ${ }^{56} \mathrm{Ni}$ and $\alpha$-particles will change depending on density and temperature. To obtain the correct values, a NSE data table provided by H.-Th. Janka and an iterative root finding algorithm were employed;

- Whenever the composition in a cell changes due to nuclear reactions, a corresponding amount of energy is added or subtracted from the total energy in that cell. The amount is derived from the nuclear binding energies published by Audi et al. (1997).

Compared to the much simpler original approach, this more detailed and realistic model has the potential to alter the explosion dynamics significantly: first, the reaction to NSE and intermediate elements with its comparatively low heat release results in lower temperature and higher density of the burned material, which will decrease the buoyancy of the burning bubbles. In addition the number of particles per unit mass in the ashes is also higher with the new model, which reduces the temperature even more. Since the $\alpha$-particles will be converted to nickel during the expansion of the star, not all of the nuclear energy is released at once when material is processed by the flame, but the release is partially delayed. The combination of all these effects is expected to shift the maximum of energy generation to a later time during the explosion.

The transition densities from burning to NSE and incomplete burning, as well as from incomplete burning to flame extinction were derived from data of a W7 run provided by K. Nomoto. This approach is rather phenomenological, and since these densities can have a potentially large impact on the simulation outcome, it will have to be re-examined in a thorough manner.

\subsection{Thin flame model}

The numerical representation of the thermonuclear reaction front (i.e. the location where the "fast" reactions take place) did not change fundamentally compared to the original implementation (Reinecke et al. 1999b). To repeat briefly, the flame front is associated with the zero level set of a function $G(r, t)$, whose temporal evolution is given by

$\frac{\partial G}{\partial t}=-\left(v_{\mathrm{u}}+s_{\mathrm{u}} n\right)(-n|\nabla G|)$,

where $v_{\mathrm{u}}$ and $s_{\mathrm{u}}$ denote the fluid and flame propagation velocity in the unburned material ahead of the front, and $n$ is 
the front normal pointing towards the fuel. The advection of $G$ caused by the fluid motions is treated by the piecewise parabolic method (Colella \& Woodward 1984), which is also used by our code to integrate the Euler equations. After each time step, the front is additionally advanced by $s_{\mathrm{u}} \Delta t$ normal to itself.

This equation is only applied in the close vicinity of the front, whereas in the other regions $G$ is adjusted such that

$|\nabla G|=1$.

The source terms for energy and composition due to the fast thermonuclear reactions in every grid cell are determined as follows:

$X_{\text {Ashes }}^{\prime}=\max \left(1-\alpha, X_{\text {Ashes }}\right)$

$X_{\text {Fuel }}^{\prime}=1-X_{\text {Ashes }}^{\prime}$

$e_{\text {tot }}^{\prime}=e_{\text {tot }}+q\left(X_{\text {Ashes }}^{\prime}-X_{\text {Ashes }}\right)$,

where $\alpha$ is the volume fraction of the cell occupied by unburned material; this quantity can be determined from the values of $G$ in the cell and its neighbours. The quantity $q$ represents the specific energy release of the total reaction.

In the new model for the nuclear reactions (see Sect. 2.1) the composition of the ashes depends on the density of the unburned material ahead of the front, which cannot be properly determined from the state variables of the grid cell in question, since it contains a mixture of burned and unburned states from which a reconstruction is not possible. Currently the maximum density of all neighbour cells is adopted as an approximation for the real density of the unburned material and used to determine the compositon of the newly created ashes. This approach is acceptable in the context of SNe Ia, since the white dwarf does not contain steep density gradients (with the exception of the flame itself), and supersonic phenomena, which might create such gradients, do not occur.

\subsection{Model for the turbulent flame speed}

All multidimensional simulations of exploding white dwarfs share the problem that it is impossible to resolve all hydrodynamically unstable scales. The consequence is that the simulated thermonuclear flame can only develop structures on the resolved macroscopic scales, while the real reaction front will be folded and wrinkled on scales down to the Gibson scale $l_{\mathrm{g}}$, which is indirectly defined by

$v^{\prime}\left(l_{\mathrm{g}}\right)=s_{1}$,

where $v^{\prime}(l)$ denotes the amplitude of velocity fluctuations on a scale $l$ and $s_{1}$ the laminar flame propagation speed. In our simulations, $l_{\mathrm{g}}$ almost always lies far below the grid scale. Simply neglecting the surface increase on sub-grid scales would lead to an underestimation of the energy generation rate, which is not acceptable; therefore a model for a turbulent flame speed $s_{\mathrm{t}}>s_{1}$ is required to compensate this effect.
For the case of very strong turbulence (i.e. for $v^{\prime}(\Delta) \gg$ $s_{1}$ or $l_{\mathrm{g}} \ll \Delta$ for a numerical resolution of $\Delta$ ) it has been shown that the turbulent flame velocity decouples from $s_{1}$ and is proportional to the velocity fluctuations $v^{\prime}$ (Shchelkin 1943; Pocheau 1992; Peters 2000). In our simulations this condition is fulfilled except at the very beginning of the explosion, where combustion starts laminar before sufficient turbulence has been generated; this stage only lasts a few tens of milliseconds. At lower densities $\left(\rho \lesssim 10^{7} \mathrm{~g} \mathrm{~cm}^{-3}\right)$ this picture is possibly not correct, since in that case the combustion takes place in the socalled distributed burning regime (Peters 1986); it is still a matter of debate whether a transition to a detonation can occur at these densities (Niemeyer 1999; Khokhlov et al. 1997), which would of course lead to a quite different chemical composition and a higher energy release in the outer stellar layers. For the time being we ignore the possibility of a delayed detonation; if further work in this field indicates that this scenario cannot be ruled out, however, this point will have to be addressed.

As discussed above, the quantity $v^{\prime}(\Delta)$ must be known in order to determine the turbulent macroscopic flame speed $s_{\mathrm{t}}$. Unfortunately it cannot be determined directly from the Reynolds stress tensor at the location of the front because of the velocity jump between burned and unburned material. Therefore we make use of a technique first presented by Clement (1993) and later applied to SNe Ia by Niemeyer \& Hillebrandt (1995), which models the creation, advection and dissipation of the turbulent kinetic energy on sub-grid scales $q$ from which $v^{\prime}(\Delta)$ can be easily derived.

The time evolution of $q$ is given by

$$
\begin{aligned}
\frac{\partial(\rho q)}{\partial t}+\nabla(\bar{v} \rho q)= & -\frac{2}{3} \rho q \nabla \bar{v}+\Sigma_{i j} \frac{\partial \bar{v}_{i}}{\partial x_{j}} \\
& -\rho e_{\text {diss }}+\nabla\left(\eta_{\text {turb }} \nabla q\right)
\end{aligned}
$$

The individual source and sink terms on the right hand side can be interpreted as turbulent compression, input from macroscopic scales, dissipation into thermal energy and turbulent diffusion, respectively.

Following Clement (1993), the turbulent viscosity, stress tensor and energy dissipation are modeled by

$\eta_{\text {turb }}=\rho \Delta v^{\prime}(\Delta)=\rho \mathcal{C} \Delta \sqrt{q}$,

$\Sigma_{i j}=\eta_{\text {turb }}\left(\frac{\partial \bar{v}_{i}}{\partial x_{j}}+\frac{\partial \bar{v}_{j}}{\partial x_{i}}-\frac{2}{3} \delta_{i j}(\nabla \bar{v})\right) \quad$ and

$e_{\mathrm{diss}}=v^{3} \Delta^{-1}=\mathcal{D} q^{3 / 2} \Delta^{-1}$.

The remaining free parameters $\mathcal{C}$ and $\mathcal{D}$ are set to

$\mathcal{C}=0.1 \mathcal{F}$ and

$\mathcal{D}=0.5 \mathcal{F}^{-1}$,

where $\mathcal{F}=\min \left(100, \max \left(0.1,10^{-4} e_{i} / q\right)\right)$,

again in accordance to Clement's work.

From the appearance of only one cell dimension $\Delta$ in the above equations it is obvious that this approach is 
only valid for grids with quadratic and cubic cells. For anisotropic grids, Clement suggests to calculate $\eta_{\text {turb }}$ separately for each spatial direction, but to treat the dissipation still as an isotropic effect. The expressions for $\eta_{\text {turb }}$ and $e_{\text {diss }}$ then become

$\eta_{\text {turb }, i}=\rho \mathcal{C} \Delta_{i}^{2} \bar{\Delta}^{-1} \sqrt{q}$ and

$e_{\text {diss }}=\mathcal{D} q^{3 / 2} \bar{\Delta}^{-1}$

where $\bar{\Delta}$ is the geometric mean of the individual cell dimensions. However, even if this extension is used, the model is expected to perform rather poorly in the extreme case of very elongated cells, since the concept of a "cell size", on which $q$ is defined, breaks down under these circumstances.

The model described above produces an approximation of the turbulent sub-grid energy throughout the computational domain, although the source and sink terms for $q$ must be disabled in the cells cut by the front because of the velocity jump. This is possible because $q$ is transported into these cells by means of advection and turbulent diffusion. The desired magnitude of the velocity fluctuations is then given by

$v^{\prime}(\Delta)=\sqrt{2 q}$,

which completes the model for the flame propagation speed.

In the given form, the sub-grid model is correct for three-dimensional simulations only; in two spatial dimensions it is necessary to replace the factor $2 / 3$ in Eqs. (8) and (10) by a factor 1 to preserve the tracelessness of $\Sigma_{i j}$. Leaving this factor unchanged would imply three-dimensional turbulent motions on unresolved scales, whereas the flow on the macroscopic scales is only twodimensional. Since the transition scale between both regions is identical to the resolution, one would expect different simulation results for identical initial conditions, if the cell sizes are varied; this is certainly not desirable. In the older versions of the simulation code this fact was overlooked, and all two-dimensional calculations were performed with the incorrect factor $2 / 3$; this resulted in a rather strong dependence of the total energy release from the numerical resolution. With the corrected model, however, the resolution does not influence the explosion energetics significantly (see Sect. 3 ).

It must be emphasized at this point that any attempt to simulate turbulent flow in two dimensions will likely produce results that differ significantly from those of fully three-dimensional simulations; this effect is caused by the unrealistic absence of turbulent velocity fluctuations along the third coordinate axis, which may have a strong influence on the scaling behaviour of the turbulent motions. Therefore the results of two-dimensional calculations involving turbulent flow must be checked at least punctually against their three-dimensional equivalents in order to give them the required credibility.

\section{Two-dimensional calculations}

The goal of the calculations presented here was to verify that variations of parameters without direct physical relevance (e.g. numerical resolution) do not affect the results significantly. Because of the enormous computing and storage requirements for this kind of numerical study, the simulations had to be performed in two dimensions assuming axisymmetry instead of solving the full threedimensional problem. Nevertheless, the insights gained from these experiments can - at least in a qualitative manner - also be applied to the three-dimensional case.

In analogy to the calculations described in Reinecke et al. (1999a), the experiments were carried out in cylindrical $(r, z)$ coordinates with a uniform grid spacing in the inner regions of the computational domain (from the origin to a radius of $\approx 2.3 \times 10^{8} \mathrm{~cm}$ ). In the outer regions the radial and axial dimensions of the grid cells were increased exponentially in order to avoid mass loss across the grid borders due to the stellar expansion. Equatorial symmetry was assumed; at the same time rotational symmetry around the polar axis was imposed by the choice of coordinates. The white dwarf was constructed by assuming a central density of $2.9 \times 10^{9} \mathrm{~g} \mathrm{~cm}^{-3}$, a uniform temperature of $5 \times 10^{5} \mathrm{~K}$ and a composition of $X_{\mathrm{C}}=X_{\mathrm{O}}=0.5$, resulting in a mass of $2.797 \times 10^{33} \mathrm{~g}$, a radius of about $1.8 \times 10^{8} \mathrm{~cm}$ and a binding energy of $5.19 \times 10^{50} \mathrm{erg}$.

The initial sub-grid energy level was set to $q=$ $10^{12} \mathrm{erg} \mathrm{g}^{-1}$, corresponding to turbulent velocity fluctuations of about $1.4 \times 10^{6} \mathrm{~cm} \mathrm{~s}^{-1}$ on the grid scale. This is a bit slower than the speed presently assumed for typical convective motions in a white dwarf before explosion (S. Woosley, personal communication), but according to the observations of Niemeyer \& Hillebrandt (1995) the large-eddy model tends to adapt itself to the macroscopic flow on a very short time scale $\left(\approx 10^{-2} \mathrm{~s}\right)$, so that the initial value for $q$ becomes largely irrelevant. This behavior was confirmed in the current simulations.

\subsection{Resolution study}

To study the robustness of our code with respect to a change of the numerical resolution, simulations were performed with grid sizes of $128^{2}, 256^{2}, 512^{2}$ and $1024^{2}$ cells, whose corresponding resolutions in the uniform inner part of the grid were $2 \times 10^{6} \mathrm{~cm}, 10^{6} \mathrm{~cm}, 5 \times 10^{5} \mathrm{~cm}$ and $2.5 \times 10^{5} \mathrm{~cm}$. The initial flame geometry (called c3_2d) used for all these calculations is identical to the setup C3 presented by Reinecke et al. (1999a): the matter within a radius of $1.5 \times 10^{7} \mathrm{~cm}$ from the stellar center was incinerated, and the surface of the burned region was perturbed to accelerate the development of Rayleigh-Taylor instabilities.

Figure 1 shows the energy release of the models; except for the run with the lowest resolution, the curves are nearly identical in the early and intermediate explosion stages. Simulation c3_2d_128 exhibits a very slow initial energy increase and does not reach the same final level 


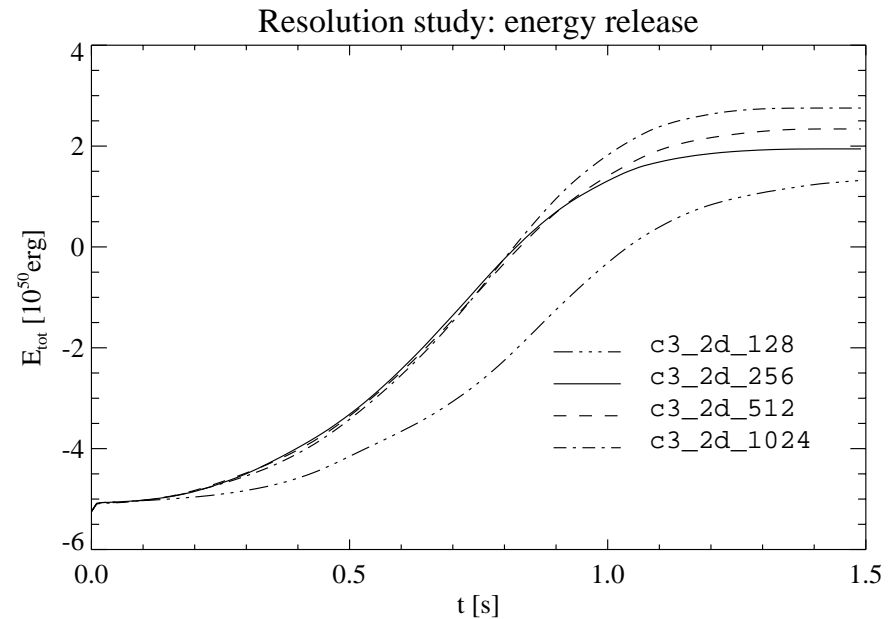

Fig. 1. Time evolution of the total energy for the initial flame geometry c3_2d and different resolutions. During the early and intermediate explosion stages there is excellent agreement between the better resolved simulations.

as the other models. Most likely this is due to insufficient resolution, which leads to a very coarsely discretized initial front geometry and thereby to an underestimation of the flame surface. From this result it can be deduced that all supernova simulations performed with our code should have a central resolution of $10^{6} \mathrm{~cm}$ or better.

After approximately $1.2 \mathrm{~s}$ the thermonuclear reactions cease and no further energy is released, although the star still contains considerable amounts of carbon and oxygen. This is explained by the rapid density drop in the ejecta caused by the bulk expansion of the star. At densities below $\approx 10^{7} \mathrm{~g} \mathrm{~cm}^{-3}$ the time scales for the strongly exothermal reactions become comparable to the expansion time scale of the white dwarf. The energy release of this late burning stage is most likely much smaller than the amount generated earlier, unless a transition to a detonation takes place (see Sect. 2.3). Moreover the description of the reaction zone as a thin flame is no longer applicable in this situation. For these reasons burning was suppressed completely at densities below $10^{7} \mathrm{~g} \mathrm{~cm}^{-3}$ (see Sect. 2.1).

In the late explosion phase (after about $0.8 \mathrm{~s}$ ) the total energy is no longer equal for the three simulations, but increases with better resolution. The origin of this effect is not clear, but since it occurs at the time when the flame enters the anisotropic part of the grid, it is probably caused by inaccuracies of the burning and hydrodynamic algorithms in the highly elongated cells in these regions. A breakdown of the sub-grid turbulence model is the most likely culprit, since in a very anisotropic grid the concept of a "grid scale" is not well defined anymore. In any case the observed scatter in the final energy releases of the order of $10 \%$ was assumed to be acceptable.

Overall, our model for the turbulent flame speed appears to compensate the lack of small structures in the front very well.

Another argument for the reliability of the turbulence model is provided by Fig. 2, which shows the front geometry and turbulence intensity after one second for the simulations c3_2d_256 and c3_2d_1024. These data are in very good agreement with the expectations:

- Both simulations have quite similar large-scale features; e.g. the burned areas appear similar in size, and the largest distance from the front to the center also is nearly equal,

- Because of the larger scale range in c3_2d_1024, the flame structure is much better resolved: it shows, for example, the onset of Kelvin-Helmholtz-instabilities in the shear flow between rising hot material and falling fuel near the coordinate axes and also small, secondary Rayleigh-Taylor instabilities, which are not visible in the coarser simulation;

- The amplitude of sub-grid fluctuations is lower for the better resolved simulation, which is in agreement with the turbulent velocity scaling law. This is also required to compensate the larger flame surface and keep the total energy generation rate resolution-independent.

This plot also demonstrates convincingly that the highest turbulence intensities are reached in the shear layer between fuel and ashes.

\subsection{Comparison to earlier $2 D$ simulations}

Comparison of the energy evolution with the results presented by Reinecke et al. (1999a) reveals that the final absolute energies are considerably higher for the refined code than for the older calculations. The remnant, which had formerly remained bound, now reaches a positive total energy and will therefore continue its expansion. However, the energy release is still too low to account for a typical SN Ia.

Significant differences also exist in the time evolution of the energy generation rate (Fig. 3): the period of most intense burning, which was originally reached after 0.2 $0.5 \mathrm{~s}$, has now shifted to $0.5-0.8 \mathrm{~s}$ after ignition. The main reason for this delayed energy release is the more realistic treatment of the fusion reactions. Since carbon and oxygen are no longer instantaneously fused to nickel, but to a NSE mixture of nickel and $\alpha$-particles, less energy is released in the early burning phases, which results in a lower temperature and higher density in the ashes. This in turn decreases the buoyancy and rising speed of the burning bubbles, leading to less shear and lower turbulent burning speeds. As a compensation, the transformation of $\alpha$-particles into nickel injects additional energy into the explosion at later times.

This argument is supported by an evolution diagram of the white dwarf's composition, shown in Fig. 4. As long as the nuclear reactions take place near the center and bulk expansion is still rather slow, nickel and $\alpha$-particles are produced at a nearly constant ratio, and the specific energy release is much lower than for synthesis of pure nickel. During the continued expansion, the equilibrium concentration for $\alpha$-particles in the NSE rapidly drops towards 

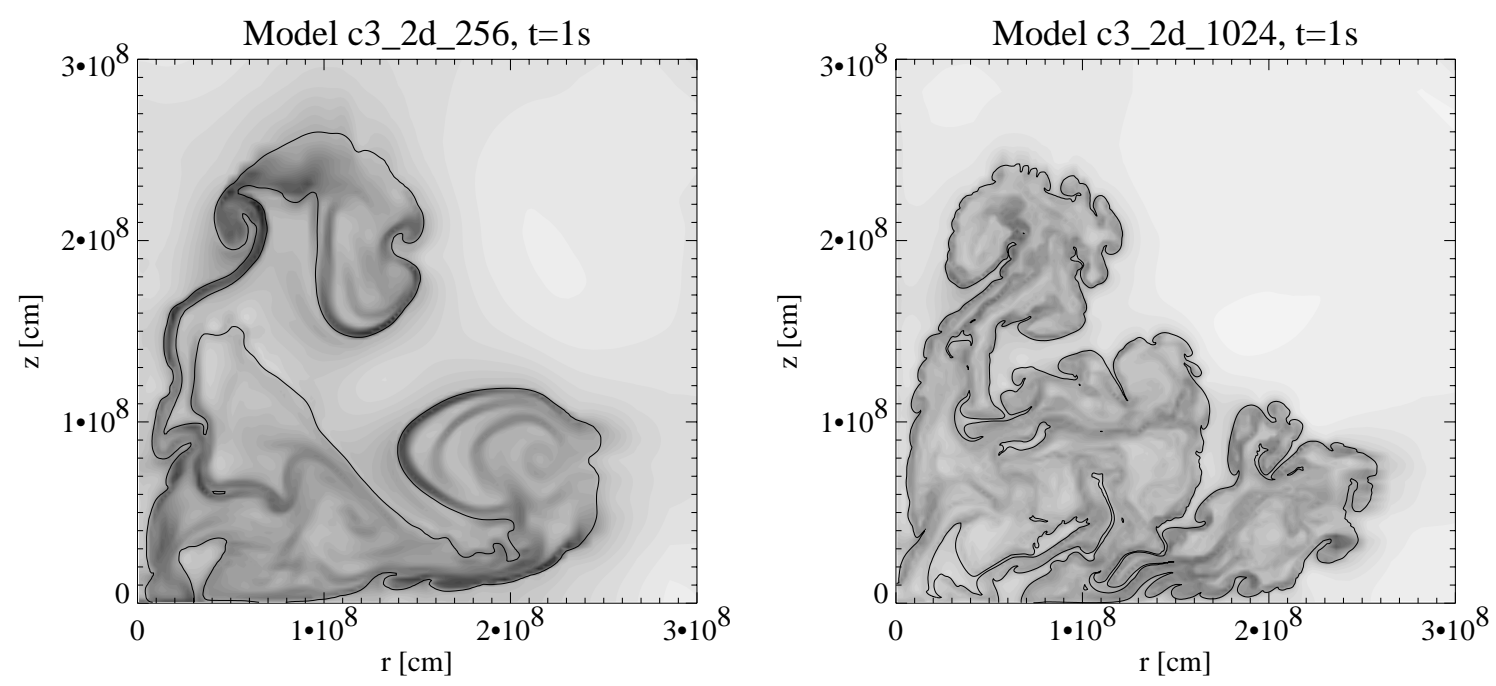

Fig. 2. Comparison of two simulations with identical initial conditions, but different resolution. The front geometry is indicated by a black line, whereas the amplitude of turbulent velocity fluctuations is given color-coded from 0 (white) to $2.5 \times 10^{7} \mathrm{~cm} \mathrm{~s}^{-1}$ (black). The low-resolution run clearly exhibits less flame structure, which is compensated by a higher flame speed. The overall features appear to be remarkably similar in both diagrams.

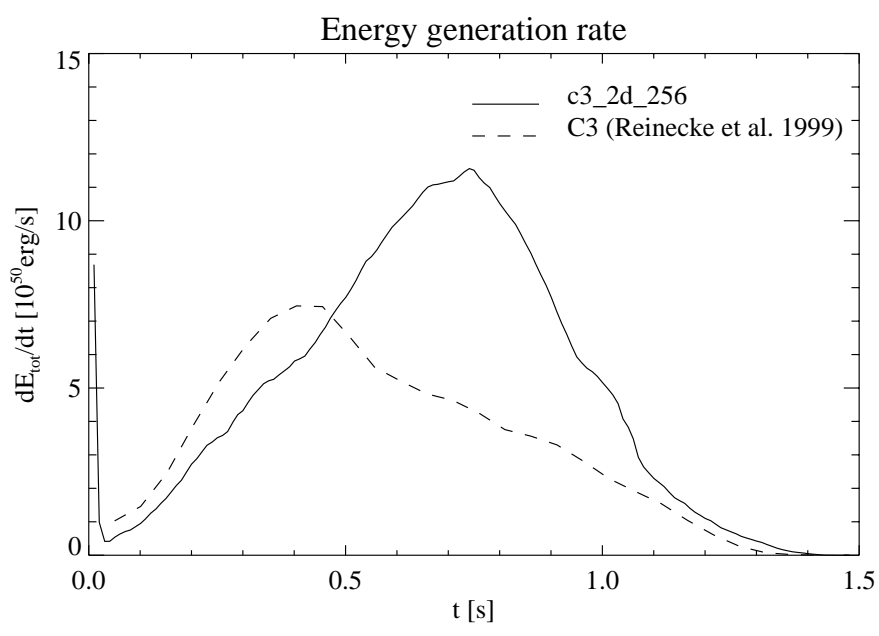

Fig. 3. Time evolution of the energy generation rate for the models C3 in Reinecke et al. (1999a) and c3_2d_265. The shift of the maximum is clearly visible.

zero and the "buffered" energy is released as thermal energy, further driving the expansion. This very exothermic process $\left(Q \approx 1.52 \times 10^{18} \mathrm{erg} \mathrm{g}^{-1}\right)$ coincides rather well with the maximum of the energy generation rate (Fig. 3).

\section{Extension to three dimensions}

In order to compare two- and three-dimensional simulations directly, a 3D calculation was performed using the same initial conditions as given in Sect. 3. For this purpose the initial two-dimensional flame location was rotated by 90 degrees around the $z$-axis and mapped onto the threedimensional Cartesian grid consisting of $256^{3}$ cells with a central resolution of $10^{6} \mathrm{~cm}$. Only one octant of the white dwarf was simulated and mirror symmetry was assumed with respect to the coordinate planes.

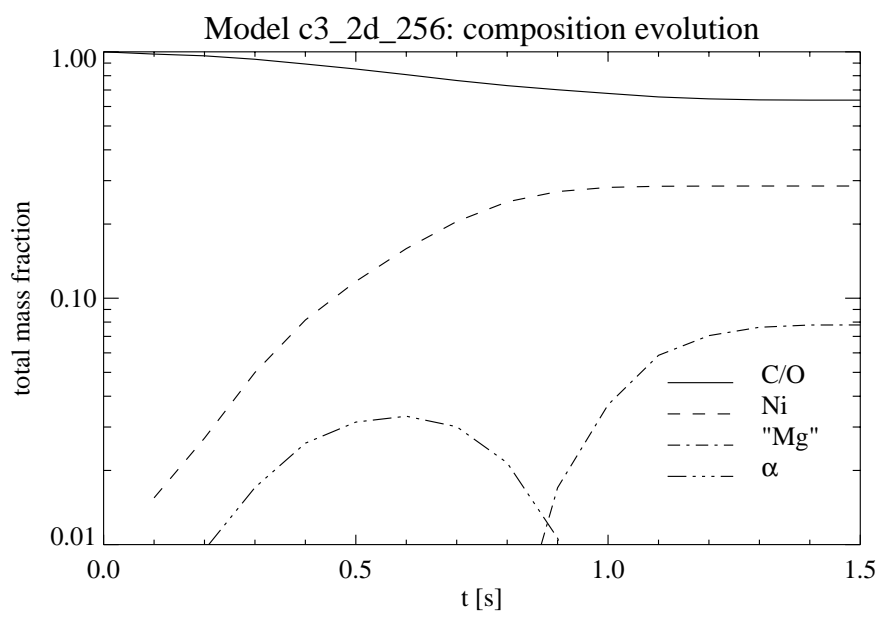

Fig. 4. Time evolution of the chemical composition for model c3_2d_256.

The initial configuration, as well as snapshots at later times, are shown in Fig. 5. Obviously, the initial axisymmetry is lost after $0.2-0.3 \mathrm{~s}$, although no explicit perturbation in $\varphi$-direction was applied to the front. This happens because the initial flame geometry cannot be mapped perfectly onto a Cartesian grid and therefore the front is not transported at exactly the same speed for all $\varphi$. On a cylindrical grid, which matches the symmetry of the problem setup better, this effect would not be observed.

In our case, however, this symmetry breaking is desired, since an exactly axisymmetric calculation would only reproduce the results of the $2 \mathrm{D}$ run. Besides, perfect rotational symmetry would never occur in reality.

During the next few tenths of a second, the small deviations cause the formation of fully three-dimensional RTmushrooms, leading to a strong convolution of the flame. As expected, this phenomenon has a noticeable influence on the explosion energetics; this is illustrated in Fig. 6. 

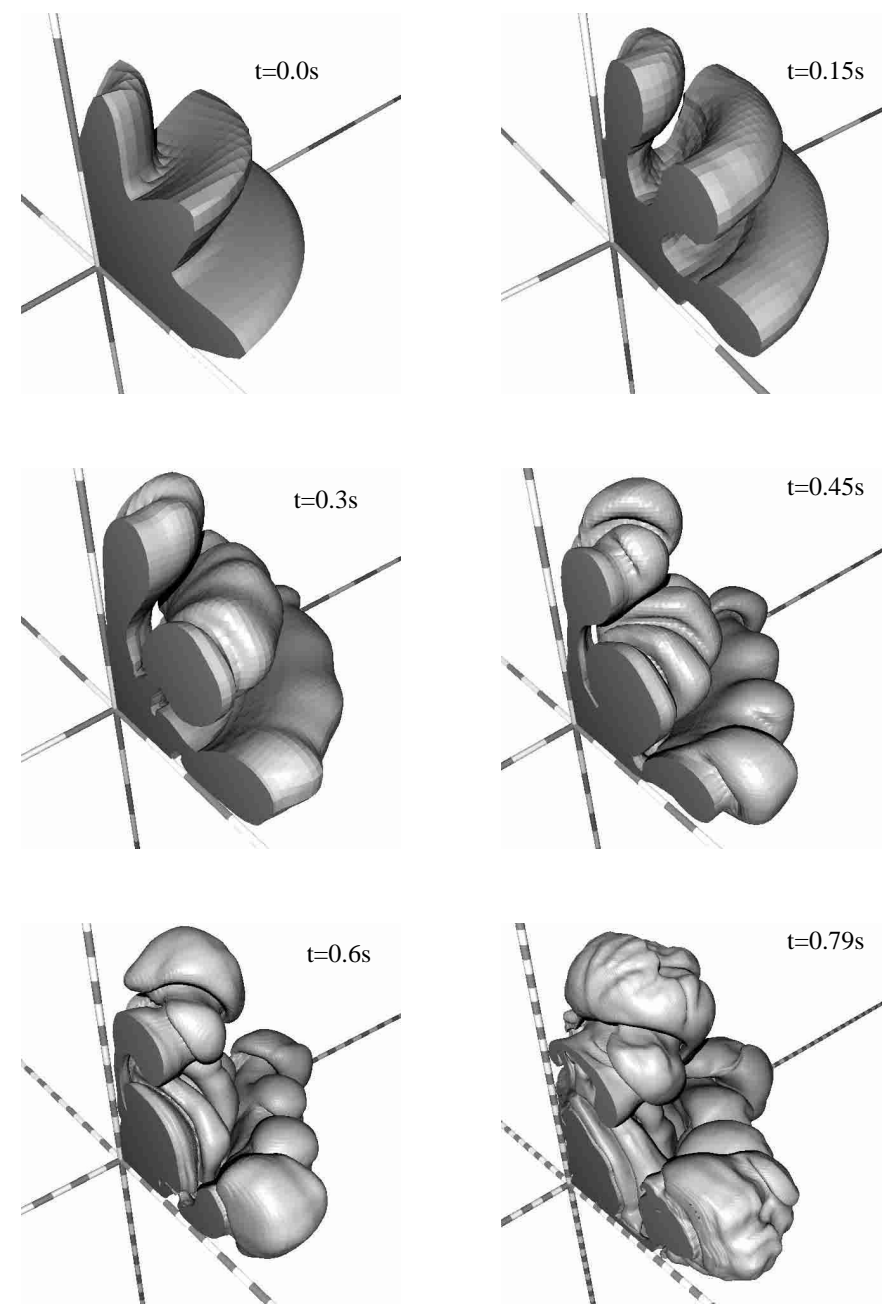

Fig. 5. Snapshots of the flame front for a centrally ignited three-dimensional scenario. One ring on the coordinate axes corresponds to $10^{7} \mathrm{~cm}$.

Before the loss of axial symmetry in c3_3d_256, the total energy evolution is almost identical for both simulations, which strongly suggests that the two- and threedimensional forms of the employed turbulence and level set models are consistent, i.e. that no errors were introduced during the extension of these models to three dimensions. In the later phases the $3 \mathrm{D}$ model releases more energy as a direct consequence of the surface increase shown in Fig. 5.

\section{Discussion and conclusions}

Since the explosion energy and the composition of the remnant are important criteria for the quality of a SN Ia model, the relevant data for all performed simulations are listed in Table 1.

Given the stellar binding energy of $5.19 \times 10^{50} \mathrm{erg}$, it is evident that the progenitor becomes unbound in all experiments, which implies that no recontraction will occur and no compact object remains. Nevertheless only model c3_3d_256 results in a powerful enough explosion to qualify as a typical SN Ia; the two-dimensional scenarios are too

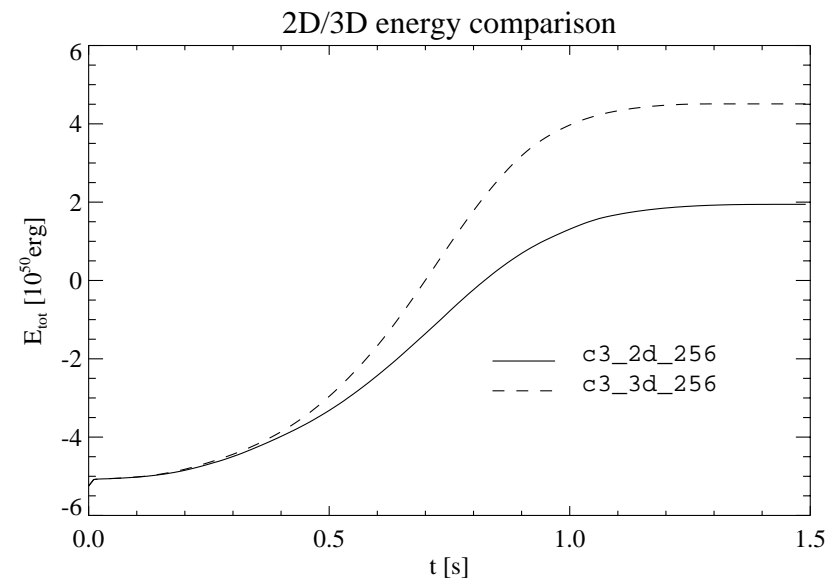

Fig. 6. Comparison of the explosion energy for identical initial conditions and resolution in two and three dimensions. After the loss of axial symmetry (at $t \approx 0.3 \mathrm{~s}$ ) the larger flame surface in the three-dimensional model leads to more vigorous burning.

Table 1. Overview over element production and energy release of all discussed supernova simulations.

\begin{tabular}{lccc}
\hline \hline model name & $\frac{m_{\mathrm{Mg}}}{M_{\odot}}$ & $\frac{m_{\mathrm{Ni}}}{M_{\odot}}$ & $\frac{E_{\text {nuc }}}{10^{50} \mathrm{erg}}$ \\
\hline c3_2d_128 & 0.132 & 0.348 & 6.56 \\
c3_2d_256 & 0.109 & 0.400 & 7.19 \\
c3_2d_512 & 0.151 & 0.402 & 7.58 \\
c3_2d_1024 & 0.152 & 0.428 & 8.00 \\
c3_3d_256 & 0.177 & 0.526 & 9.76 \\
\hline
\end{tabular}

weak to accelerate the ejecta to the speeds observed in real events and produce too little nickel to power a standard SN Ia light curve, which had more or less to be expected, since the surface growth was partially suppressed.

The 3D calculation is a good candidate for typical SN Ia explosions, at least with respect to explosion strength and remnant composition. Its nickel mass falls well into the range of $\approx 0.45-0.7 M_{\odot}$ determined by Contardo et al. (2000) for several typical events, and it can be deduced from the amount of "magnesium" in the ejecta that enough intermediate mass elements were synthesized to explain the observed spectral features.

Qualitatively, our results for the explosion energetics are in rather good agreement with recent simulations performed by Khokhlov (2000), which rely on quite different numerical models and assumptions; most notably the macroscopic flame velocity is determined by the asymptotic rise speed of a Rayleigh-Taylor bubble with size $\Delta$, instead of the turbulent velocity fluctuations. The particular setup described in his paper is centrally ignited, and no perturbation is applied to the spherical flame surface. Due to the high resolution the discretization errors are quite small, and there is a rather long phase of very slow burning until the first instabilities have reached the nonlinear stage; from this moment on, however, the explosion progresses in a way very similar to the $3 \mathrm{D}$ simulation discussed here. This similarity applies to the energy 
production rate as well as the geometrical features developed by the flame.

Further improvements of the SN Ia simulations are planned, both with respect to the underlying models and the realism of the initial conditions. As was pointed out by S. Blinnikov (personal communication), an accurate simulation of light curves and spectra based on the results of the 3D hydrodynamical computation requires not only the total concentration of iron group elements (which have been represented so far by ${ }^{56} \mathrm{Ni}$ ), but the amounts of the real ${ }^{56} \mathrm{Ni}$ and the rest of the Fe-group elements. The necessary changes will be implemented in the near future.

It would also be desirable to reach a higher numerical resolution at the beginning of the explosion, which allows a more accurate prescription of the flame geometry, and, at the same time, to follow the expansion of the star over a longer time period (several seconds), until the ballistic expansion stage has been reached. To achieve both of these goals, an expanding grid must be used instead of the currently employed static one.

Acknowledgements. The authors thank Ken'ichi Nomoto for pointing out the necessity to incorporate the NSE effects into our burning model and for providing W7 datasets, and Hans-Thomas Janka, who provided the required NSE data tables.

This work was supported in part by the Deutsche Forschungsgemeinschaft under Grant Hi 534/3-3. The numerical computations were carried out on a Hitachi SR-8000 at the Leibniz-Rechenzentrum München as a part of the project H007Z.

\section{References}

Audi, G., Bersillon, O., Blachot, J., \& Wapstra, A. H. 1997, Nuclear Phys. A, 624, 1

Clement, M. J. 1993, ApJ, 406, 651

Colella, P., \& Woodward, P. R. 1984, J. Comput. Phys., 54, 174

Contardo, G., Leibundgut, B., \& Vacca, W. D. 2000, A\&A, 359,876

Khokhlov, A. M. 2000, Three-Dimensional Modeling of the Deflagration Stage of a type Ia Supernova Explosion, ApJ, submitted [astro-ph/0008463]

Khokhlov, A. M., Oran, E. S., \& Wheeler, J. C. 1997, ApJ, 478,678

Niemeyer, J. C. 1999, ApJ, 523, L57

Niemeyer, J. C., \& Hillebrandt, W. 1995, ApJ, 452, 769

Peters, N. 1986, in 21st Symp. on Combustion (Pittsburgh: The Combustion Institute), 1231

Peters, S. 2000, Turbulent Combustion (Cambridge: Cambridge University Press)

Pocheau, A. 1992, Europhys. Lett., 20, 401

Reinecke, M. A., Hillebrandt, W., \& Niemeyer, J. C. 1999a, A\&A, 347, 739

Reinecke, M. A., Hillebrandt, W., Niemeyer, J. C., Klein, R., \& Gröbl, A. 1999b, A\&A, 347, 724

Shchelkin, K. I. 1943, J. Tech. Phys. USSR, 13, 520 